www.jmscr.igmpublication.org

Impact Factor 5.84

Index Copernicus Value: 71.58

ISSN (e)-2347-176x ISSN (p) 2455-0450

crossref DOI:_https://dx.doi.org/10.18535/jmscr/v5i9.92

Journal Of Medical Science And Clinical Research

IGM Publication

An Official Publication of IGM Publication

\title{
Parasitic Leiomyoma an Interesting Incidental Finding with Enigmatic Origin- A Case Report
}

\author{
Authors \\ Priyanka Lad ${ }^{1}$, Mithila B. ${ }^{2}$, Atul Jain ${ }^{3}$, Hoogar M.B. ${ }^{4}$, Reeta Dhar ${ }^{5}$, Evith Pereira ${ }^{6}$, \\ Kalyani Mahore $^{7}$ and Dr Vaidehee Naik ${ }^{8}$ \\ ${ }^{1,2,8}$ Junior Resident Post-Graduate Students, ${ }^{3}$ Assistant Professor, ${ }^{4}$ Associate Professor \\ ${ }^{5}$ Professor and Head of the Department and ${ }^{6,7}$ Senior Resident Post-Graduate Students \\ Department of Pathology, MGM Medical College, Navi Mumbai, India \\ Corresponding Author \\ Dr Priyanka Lad \\ Resident, Department of Pathology, MGM Medical College, Navi Mumbai, India \\ Email: priyulad@yahoo.com, Ph no-8425800135
}

\begin{abstract}
Background: Uterine leiomyoma, a benign tumour of smooth muscle, is the most common neoplasm of the female genital tract of reproductive age group. Leiomyomas arise from smooth muscle cells of uterus and rarely from smooth muscle cells of blood vessels or dermal adnexal structures and otherorgans of gastrointestinal system such as stomach and intestines ${ }^{[1]}$.

The case being presented here is rare and interesting variant of leiomyoma observed in clinical practice, based on its growth pattern, often referred as parasitic leiomyoma, which is defined asas leiomyoma that has, due to poorly understood reasons, become partially or almost completely separated from the uterus to become autonomous to receive its main blood supply from another source ${ }^{[2]}$. A pedunculated leiomyoma arising from serosal surface of uterus develops a long stalk or is freely mobile in the parametrial or pelvic region is referred to as 'wandering' or 'migrating leiomyoma'.

Keywords: Leiomyoma, parasitic leiomyoma, smooth muscle tumour, uterus.
\end{abstract}

\section{INTRODUCTION}

Uterine leiomyoma are one of the most common neoplasms found in the female genital tract in females of reproductive age group. Clinically leiomyomas affect $20-30 \%$ of females by the age of 35 years. Most commonly leiomyomasarise from smooth muscle cells of uterus and rarely from stomach, bowel, or blood vessel wall ${ }^{[1]}$.

One of the rare and interesting growth variant of leiomyoma observed in clinical practice is of parasitic leiomyoma. Kelly and Cullen in 1909 has described parasitic leiomyoma as leiomyoma that has, for some reason become partially or almost completely separated and from the uterus to become autonomous to receive its main blood supply from another source ${ }^{[2]}$.

Leiomyomas are classified according to morphological and growth patterns. The different variants of growth pattern of leiomyoma are benign metastasizing leiomyoma, disseminated peritoneal leiomyomatosis, intravenous leiomyomatosis, parasitic leiomyoma and retroperitoneal mass ${ }^{[3]}$. 
Subserosal leiomyomas which are located in the subserosal surface of uterus can be pendunculated. If pedunculated subserosal leiomyoma may develop a stalk and may acquire a large size to be relatively freely mobile in the parametrial or pelvic region. Such leiomyoma is called as "wandering or migrating Leiomyoma". It is clinically known that such leiomyoma could get detached from stalk and get attached to omentum or broad ligament and develop auxillary blood supply from the source of its new attachment. Such a leiomyoma which becomes autonomous by detachment from its original site of origin and gets blood supply from other tissues elsewhere is called as "Parasitic Leiomyoma"[3].

\section{CASE REPORT}

A middle-aged woman, 52 years, presented to Gynaecology out-patient department with complaints of abdominal pain and distension since 6 months. Ultrasonography revealed auterine adnexal mass measuring $11.2 \times 10.2 \times 7.9 \mathrm{~cm}$. Further investigations such as Magnetic Resonance Imaging (MRI) showed solid and cystic adnexal lesion with multiple solid-cystic areas, most probably arising from ovary suggestive of mucinous cyst adenocarcinoma.

Intra-operatively, it was noted that a single, greybrown soft to firm mass measuring $14 \times 13 \times 8 \mathrm{~cm}$, which was attached to mesentery. External surface was irregular, nodular with few thin-walled blood vessels seen over it. Cut surface showed solid, grey white, lobulated mass with areas of haemorrhage and cystic change. Multiple cystic areas containing yellowish coloured fluid [Figure $1 \mathrm{~A}$ and $\mathrm{B}$, and Figure $2 \mathrm{~A}$ and $\mathrm{B}$ ] were noted. The serosal surface of the uterus and both the ovaries did not show any topographic or spatial relationship with the mass which was apparently arising from the mesentery.

Microscopic examination revealed a wellcircumscribed lesion composed of interlacing fascicles of smooth muscle cells arranged in whorled pattern and in irregular fascicles at places interspersed by scant fibrous tissue with diffuse areas of hyaline change. At places, the fascicles of smooth muscles appeared markedly oedematous with consequent rarefaction of smooth muscle fibres [Figure 3A, 10x and B, 40x].

The sections from the lesion which were stained with various immunohistochemical markers were diffusely positive for smooth muscle actin (SMA) and Desmin, the latter showing diffuse positivity in cellular areas; sections were also diffusely positive for vimentin while being diffusely negative for epithelial immunohistochemical markers such as EMA and Pancytokeratin.

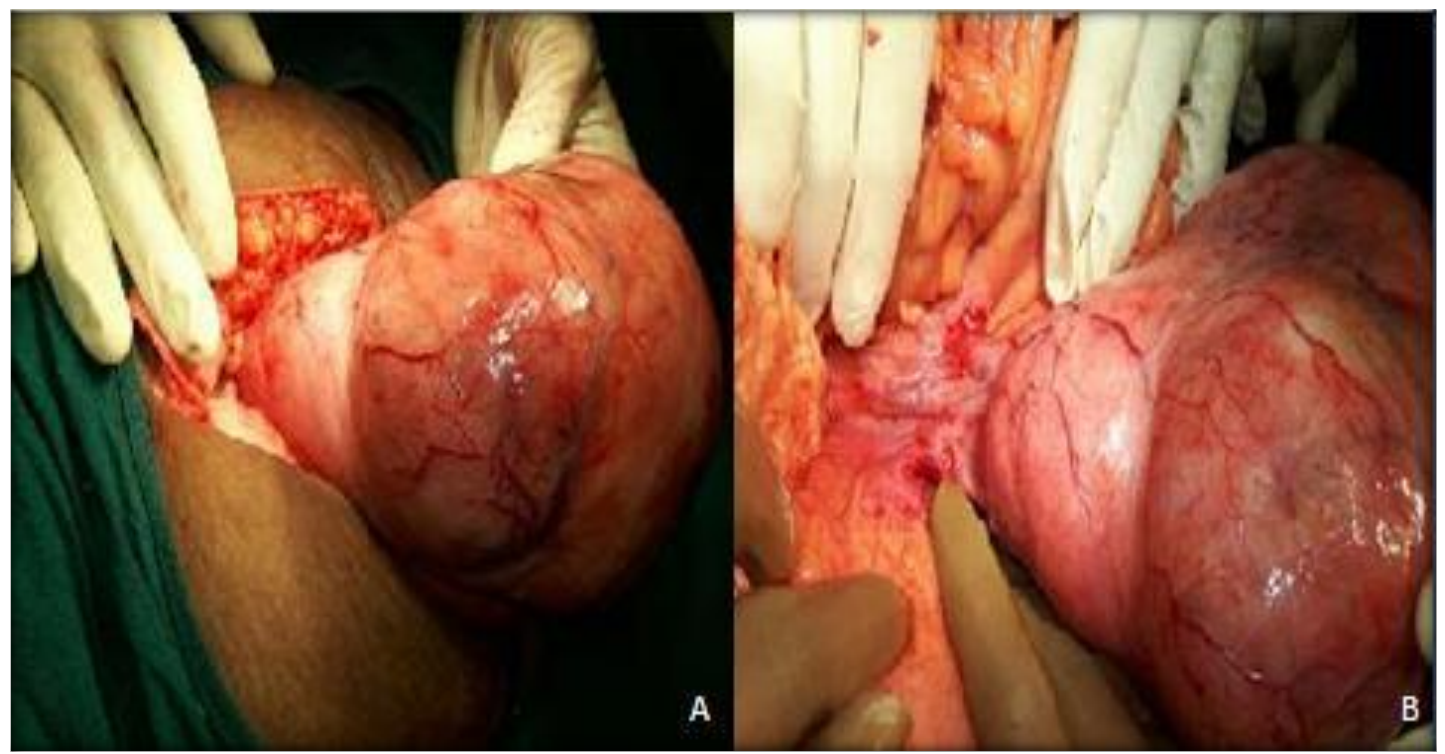

Figure 1 -A and B: Photographs Showing intra-operative images of adnexal mass attached to mesentry. 


\section{JMSCR Vol||05||Issue||09||Page 27897-27901||September}

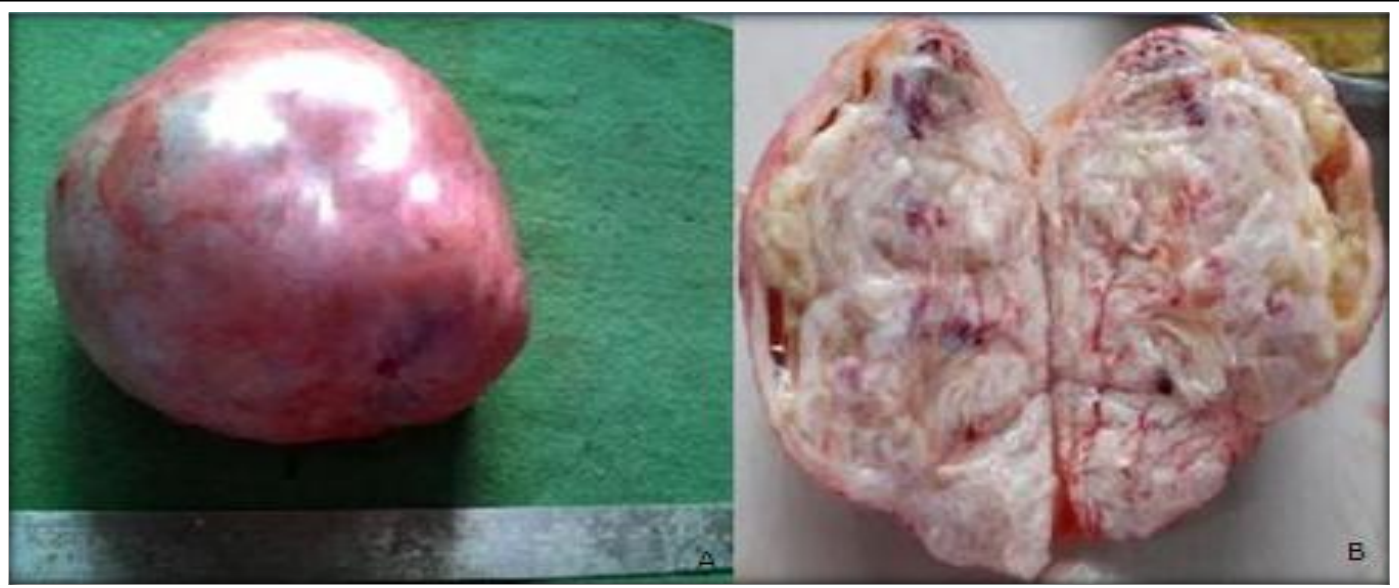

Figure 2-A: Photograph Showing greyish white globular mass measuring 14x 13x8cm.

Figure 2-B: Cut section shows solid, grey-white mass with focal cystic and haemorrhagic areas.

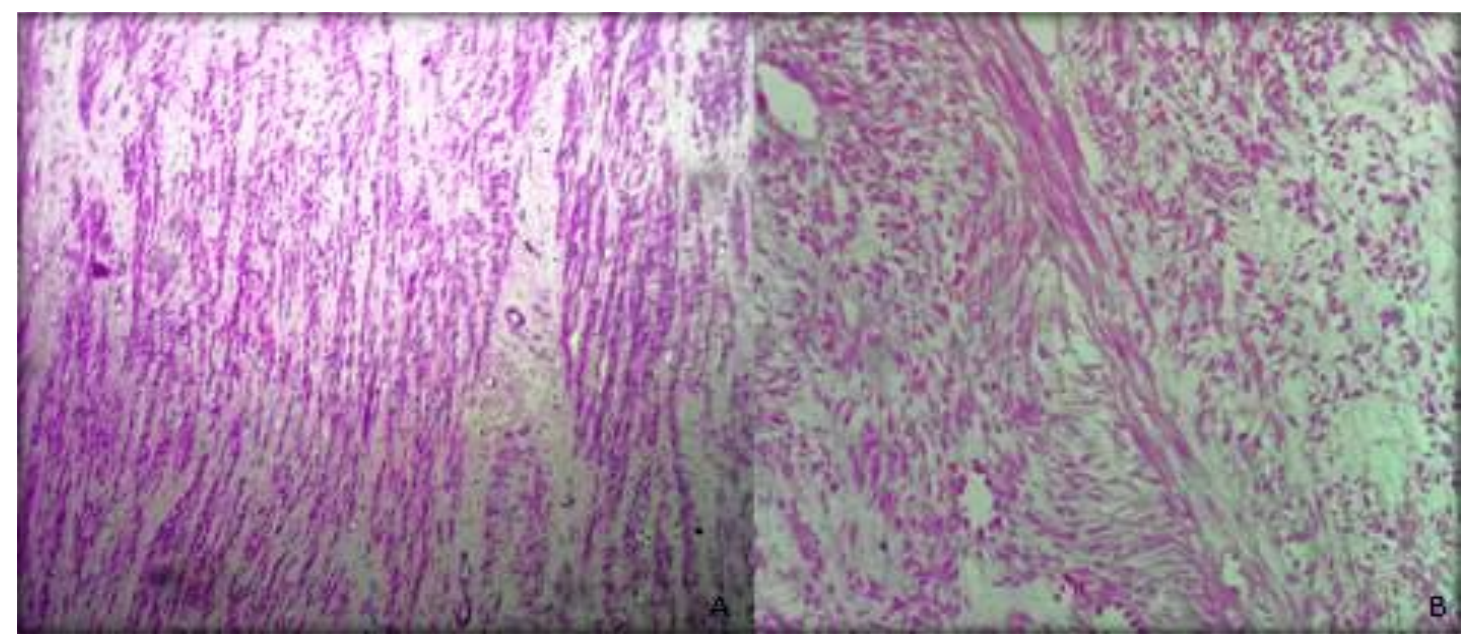

Figure 3-A and 3-B. Photomicrographs showing intermingling fascicles of smooth muscle (Figure 3-A); Smooth muscle fascicles show marked oedema with rarefaction of smooth muscle fibre at places (Figure 3B).

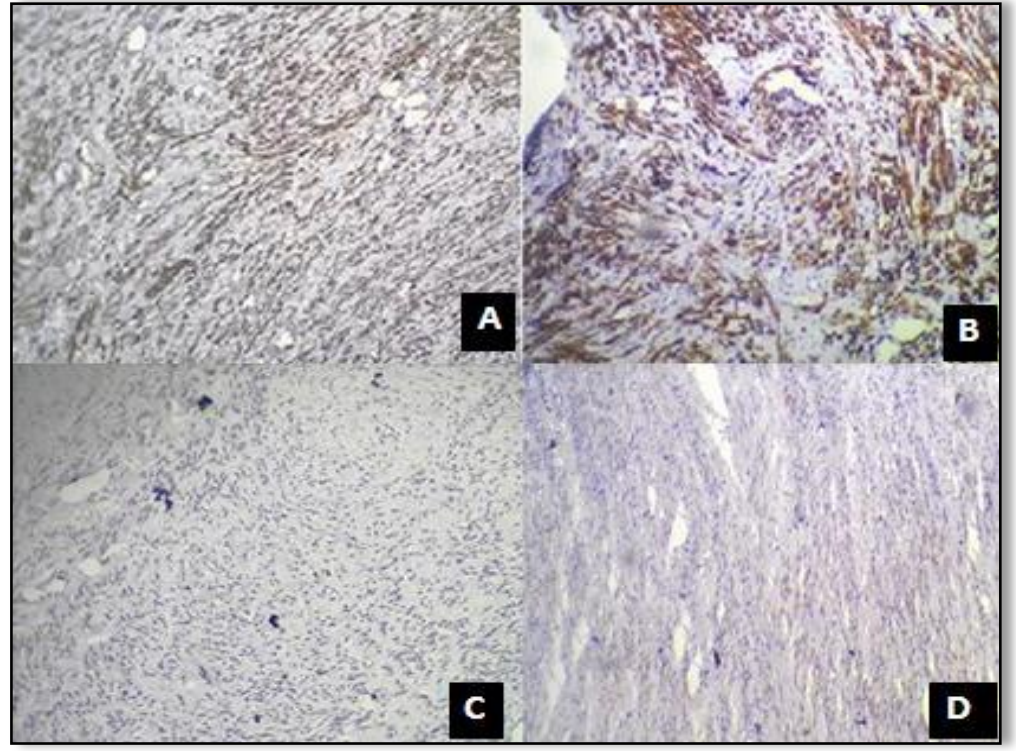

Figure 4-A Photomicrograph Showing tumour cells showing diffuse positivity for Vimentin. B: Photomicrographs Showing diffuse positivity for immunohistochemical markers for smooth muscle actin.C and D shows Ck and S-100 Negativity. 


\section{DISCUSSION}

Parasitic leiomyomas, which are rare growth variants of benign tumours of smooth muscle occur as extra-uterine tumours. The origin of parasitic leiomyomas is often enigmatic, though two theories are proposed for their occurrence by Scully and Cullen, who incidentally happen to be first to coin the term 'parasitic leiomyoma, which later on came to be known as 'wandering leiomyoma', 'migratory leiomyoma', and 'ectopic leiomyoma ${ }^{,[2]}$. According to one of the theories, parasitic leiomyomas are a rare variant of pedunculated subserosal leiomyomas that have outgrown their uterine blood supply and become separated from uterus and develop new sources of blood supply from another adjacent unrelated tissue structure or organ. Second theory proposes that parasitic leiomyomas could be "iatrogenic" in their origin and they may be caused by seeding of portions of leiomyomas during myomectomy or hysterectomy. Surgical procedures using morcellation technique could lead to possibility of inadvertent leaving behind of a tiny piece of leiomyoma leading to its implantation in any part of the body cavity to grow as autonomous mass later. The fact this morcellation could lead to remnant of an excised leiomyoma left behind growing into a parasitic leiomyoma should always be considered by surgeon in the differential diagnoses of nodular masses in the pelvic cavity or parametrial region ${ }^{[2-6]}$. Other suggested possibilities that metaplasia of pluripotent cells could be source of parasitic leiomyoma in conditions such as intravascular leiomyomatosis, benign metastasizing leiomyomatosis and de novo formation of leiomyomatosis peritonealis disseminata. ${ }^{[2]}$. In other cases, the origin of leiomyomas in other sites such as abdominal wall are not clear and it is hypothesized that such leiomyomas could be arising from that transformation of the various cells of the vessel wall in the anterior abdominal layer to leiomyoma could occur possibly due to somatic mutations and interplay of hormonal and growth factors ${ }^{[7,8]}$.

\section{CONCLUSION}

Parasitic leiomyomas rare lesions which when occur at unusual places such as body cavities could pose problems to the clinicians unless they are included in the differential diagnoses. The case reported here is unique and interesting inasmuch as it presented as ovarian mass with multiple solid and cystic areas mimicking a mucinous cyst adenocarcinoma and the origin of which was enigmatic in its origin being traced to mesentery with no spatial or topographic connection or relation to uterine wall or ovaries as both uterine wall including its serosal surface and both the ovaries were clear of any such neoplastic growth.

\section{REFERENCES}

1. Sujatha, M, Mamatha, S, Poornima, M, Srinivas, R. , Roy, P. and Jasmin, K. (2014) Parasitic Leiomyoma PeritoneumA Rare Case. Open Journal of Obstetrics and Gynecology, 4, 864-867. doi:10.4236/ojog.2014.414121.

2. Kho Kimberly, A. and Nezhat Ceana, M.D. (2009) Obstetrics and Gynecology, 114, 3.

3. Amrit Nasta, Kunal Nandy, and Yogesh Bansod, "An Unusual Case of Abdominal Leiomyoma Presenting as a Free Lying Intraperitoneal Mass in an Elderly Gentleman," Case Reports in Surgery, vol. 2016, Article ID 1714958, 3 pages, 2016. doi:10.1155/2016/1714958

4. Rajiv Mahendru, Geetinder Gaba, Shweta Yadav, Gurmeet Gaba, and Chinky Gupta, "A Rare Case of Retroperitoneal Leiomyoma," Case Reports in Surgery, vol. 2012, Article ID 425280, 3 pages, 2012. doi: $10.1155 / 2012 / 425280$

5. Kale T, Tambe S, Alnur Y. Parasitic fibroid: a diagnostic dilemma. The New Indian Journal of OBGYN. 2016; 3(1): 4750. doi:10.21276/obgyn.2016.3.1.9

6. Sreelatha S, Kumar A, Nayak V, Punneshetty S, Hanji N. A rare case of 
primary parasitic leiomyoma. Int J Reprod Contracept Obstet Gynecol 2013:2:422-4

7. Manojit Midya, Neeraj K. Dewanda, Primary Anterior Abdominal Wall Leiomyoma- A Diagnostic Enigma.Journal of Clinical and Diagnostic Research.

8. Spanoudaki, A, Oikonomou, A, Dimitrova, K., \& Prassopoulos, P. (2008). Pedunculated uterine leiomyoma mimicking abdominal mass: a case report.Cases Journal, $\quad 1$, 315. http://doi.org/10.1186/1757-1626-1-31 